\title{
Enantioselective Synthesis of Quaternary Stereocenters via a Catalytic Asymmetric Stetter Reaction
}

\author{
Mark S. Kerr and Tomislav Rovis* \\ Department of Chemistry, Colorado State University \\ Fort Collins, Colorado 80523
}

\section{Supporting Information}

General Methods. All reactions were carried out under an atmosphere of argon in flame-dried glassware with magnetic stirring. Tetrahydrofuran, diethylether, and dichloromethane were degassed with argon and passed through two columns of neutral alumina. Toluene was degassed with argon and passed through one column of neutral alumina and one column of Q5 reactant. Column chromatography was performed on EM Science silica gel 60 (230-400 mesh). Thin layer chromatography was performed on EM Science $0.25 \mathrm{~mm}$ silica gel $60-\mathrm{F}$ plates. Visualization was accomplished with UV light, or $\mathrm{KMnO}_{4}$ followed by heating.

KHMDS was purchased from Aldrich Chemical Co. as a $0.5 \mathrm{M}$ solution in toluene and used without purification. Racemic material was prepared by the method of Ciganek. ${ }^{1}$

Melting points were measured with a MelTemp II melting point apparatus outfitted with a Fluke 51 thermocouple and are uncorrected. Infrared spectra were obtained on a Nicolet Avatar 320 FT-IR spectrometer. ${ }^{1} \mathrm{H}$ NMR and spectra were recorded on a Varian 300 or $400 \mathrm{MHz}$ spectrometer at ambient temperature. Data are reported as follows: chemical shift in parts per million $(\square, \mathrm{ppm})$ from an internal standard (tetramethylsilane $[\mathrm{TMS}]$ or deuterated chloroform $\left.\left[\mathrm{CDCl}_{3}\right]\right)$, multiplicity ( $\mathrm{s}=$ singlet, $\mathrm{d}=$ doublet, $\mathrm{t}=$ triplet, $\mathrm{q}=$ quartet, $\mathrm{m}=$ multiplet, and $\mathrm{bs}=$ broad singlet), integration, and coupling constant $(\mathrm{Hz}) .{ }^{13} \mathrm{C}$ NMR were recorded on a Varian 75 or $100 \mathrm{MHz}$ spectrometer at ambient temperature. Chemical shifts are reported in ppm from $\left(\mathrm{CDCl}_{3}\right)$ taken as $77.0 \mathrm{ppm}$. Mass spectra were obtained on Fisons VG Autospec. Analytical high performance liquid chromatography (HPLC) was performed on a Dynamax model SD200 HPLC equipped with a Dynamax model UV-1 variable wavelength UV detector using a Chiracel OD-H, AD, or OB-H $(0.46 \mathrm{~cm} \mathrm{X} 25 \mathrm{~cm})$ chiral column. Gas chromatography was performed on a Varian Cp 3800 gas chromatograph equipped with a flame ionization detector using a Chiraldex B-DM or Chiraldex B-PH capillary gas chromatography column. Optical rotations were measured on an Autopol III automatic polarimeter in a $1 \mathrm{dm}$ cell.

General procedure for the asymmetric intramolecular Stetter reaction of aromatic substrates: A flame-dried round bottom flask was charged with triazolium salt $(0.02$

\footnotetext{
${ }^{1}$ Ciganek, E. Synthesis 1995, 1311.
} 
mmol, 0.2 eq) and evacuated for 5 minutes, then covered with argon. Substrate (0.1 mmol, $1 \mathrm{eq})$ was added in toluene $(1 \mathrm{ml})$ via syringe, followed by addition of triethylamine $(0.2 \mathrm{mmol}, 2 \mathrm{eq}$ to substrate) via syringe and the solution was stirred at ambient temperature under argon for 24 hours. The reaction was then poured onto a column of silica gel and eluted with a suitable solution of ethyl acetate in hexanes, to afford analytically pure product.

General procedure for the asymmetric intramolecular Stetter reaction of aliphatic substrates: A flame-dried round bottom flask was charged with triazolium salt $(0.02$ mmol, $0.2 \mathrm{eq})$ and toluene $(1 \mathrm{ml})$ under argon. To this solution was added KHMDS ( $0.5 \mathrm{M}$ in toluene) $(0.02 \mathrm{mmol}, 0.2 \mathrm{eq})$ via syringe and the solution was stirred at ambient temperature for 5 minutes. Substrate $(0.01 \mathrm{mmol}, 1 \mathrm{eq})$ was added in toluene $(1 \mathrm{ml})$ via syringe and allowed to stir for $24 \mathrm{~h}$ at ambient temperature. The reaction was then poured onto a column of silica gel and eluted with a suitable solution of ethyl acetate in hexanes, to afford analytically pure product.

(R)-(2-Ethyl-3-oxo-2,3-dihydro-benzofuran-2-yl)-acetic acid methyl ester (6):

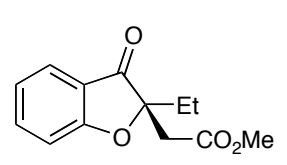
According to the general procedure, aldehyde $4(24.0 \mathrm{mg}, 0.10 \mathrm{mmol})$ in toluene $(1 \mathrm{~mL})$ was added to catalyst $5 \mathrm{c}(9.0 \mathrm{mg}, 0.02 \mathrm{mmol})$ followed by addition of triethylamine $(0.029 \mathrm{~mL}, 0.20 \mathrm{mmol})$ and allowed to stir at ambient temperature for 24 hours. Flash column chromatography (6:1 hexanes to ethyl acetate) provided $23.0 \mathrm{mg}(96 \%)$ of $\mathbf{6}$ as a clear oil: $\operatorname{Rf}(3: 1$ hexanes to ethyl acetate $)=0.24 ;[\square]_{\mathrm{D}}{ }^{25}=-74^{\circ}\left(\mathrm{CH}_{2} \mathrm{Cl}_{2}\right) ;$ HPLC analysis Chiracel OD-H column, 97:3 hexanes to isopropanol $1.0 \mathrm{ml} / \mathrm{min}$. Major enantiomer: 20.4 minutes, minor enantiomer: 32.6 minutes; ${ }^{1} \mathrm{H}$ NMR $\left(300 \mathrm{MHz}, \mathrm{CDCl}_{3}\right) \square 7.69$ (dd, $1 \mathrm{H}, J=8.4,1.5 \mathrm{~Hz}), 7.59(\mathrm{ddd}, 1 \mathrm{H}, J=8.1,7.1,1.8 \mathrm{~Hz}), 7.05-7.10(\mathrm{~m}, 2 \mathrm{H}), 3.51(\mathrm{~s}$, $3 \mathrm{H}), 3.02(\mathrm{~d}, 1 \mathrm{H}, J=16.0 \mathrm{~Hz}), 2.94(\mathrm{~d}, 1 \mathrm{H}, J=16.0 \mathrm{~Hz}), 1.89(\mathrm{q}, 2 \mathrm{H}, J=7.3 \mathrm{~Hz}), 0.83$ $(\mathrm{t}, 3 \mathrm{H}, J=7.3 \mathrm{~Hz}) ;{ }^{13} \mathrm{C} \mathrm{NMR}\left(75 \mathrm{MHz}, \mathrm{CDCl}_{3}\right) \square 202.8,171.8,169.2,137.9,124.3$, 122.0, 113.1, 89.2, 76.7 52.1, 40.6, 30.0, 7.7; IR (NaCl, neat) 2974, 2962, 1721, 1614, $1464 \mathrm{~cm}^{-1}$; HRMS (EI+) calcd for $\mathrm{C}_{13} \mathrm{H}_{14} \mathrm{O}_{4}, 234.0892$. Found 234.0887.

(R)-(5-Bromo-2-ethyl-3-oxo-23-dihydro-benzofuran-2-yl)-acetic acid methyl ester

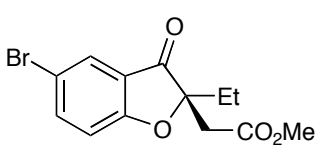

(8): According to the general procedure, aldehyde $7(37.0 \mathrm{mg}$, $0.123 \mathrm{mmol})$ in toluene $(1 \mathrm{~mL})$ was added to catalyst $\mathbf{5 c}(12.0 \mathrm{mg}$, $0.025 \mathrm{mmol}$ ) followed by addition of triethylamine $0.036 \mathrm{~mL}$ $(0.25 \mathrm{mmol})$ and allowed to stir at ambient temperature for 24 hours. Flash column chromatography (6:1 hexanes to ethyl acetate) provided $34.0 \mathrm{mg}$ $(92 \%)$ of 8 as a clear oil: $\operatorname{Rf}(4: 1$ hexanes to ethyl acetate $)=0.38 ;[\square]_{\mathrm{D}}{ }^{25}=-44^{\circ}\left(\mathrm{CH}_{2} \mathrm{Cl}_{2}\right)$; HPLC analysis - Chiracel OD-H column, 90:10 hexanes to isopropanol $1.0 \mathrm{ml} / \mathrm{min}$. Major enantiomer: 9.0 minutes, minor enantiomer: 27.6 minutes; ${ }^{1} \mathrm{H}$ NMR $(400 \mathrm{MHz}$, $\left.\mathrm{CDCl}_{3}\right) \square 7.76(\mathrm{~d}, 1 \mathrm{H}, J=2.1 \mathrm{~Hz}), 7.63(\mathrm{dd}, 1 \mathrm{H}, J=8.7,2.1 \mathrm{~Hz}), 6.97(\mathrm{~d}, 1 \mathrm{H}, J=8.7$ $\mathrm{Hz}), 3.50$ (s, 3H), $3.01(\mathrm{~d}, 1 \mathrm{H}, J=16.6 \mathrm{~Hz}), 2.94(\mathrm{~d}, 1 \mathrm{H}, J=16.6 \mathrm{~Hz}), 1.83$ (q, 2H, $J=$ $7.5 \mathrm{~Hz}), 0.80(\mathrm{t}, 3 \mathrm{H}, J=7.5 \mathrm{~Hz}) ;{ }^{13} \mathrm{C} \mathrm{NMR}\left(100 \mathrm{MHz}, \mathrm{CDCl}_{3}\right) \square 201.6,170.5,169.2$, $140.4,126.9,123.9,114.9,114.5,90.1,52.1,40.5,30.0,7.5$; IR (NaCl, neat) 2973, 1726, $1608,1464,1272,1171 \mathrm{~cm}^{-1}$. 
(R)-(2-Ethyl-3-oxo-2,3-dihydro-benzo[b]thiophen-2-yl)-acetic acid methyl ester (9):<smiles>CCC1(CC(C)=O)Sc2ccccc2C1=O</smiles>

According to the general procedure, aldehyde $9(20.0 \mathrm{mg}, 0.080$ $\mathrm{mmol})$ in toluene $(1 \mathrm{~mL})$ was added to catalyst $\mathbf{5 c}(7.5 \mathrm{mg}, 0.016$ $\mathrm{mmol})$ followed by addition of triethylamine $(0.023 \mathrm{~mL}, 0.16 \mathrm{mmol})$ and allowed to stir at ambient temperature for 24 hours. Flash column chromatography (6:1 hexanes to ethyl acetate) provided $19.0 \mathrm{mg}$ (95\%) of $\mathbf{1 0}$ as a white crystalline solid: $\operatorname{Rf}(3: 1$ hexanes to ethyl acetate $)=0.26 ;[0]_{\mathrm{D}}{ }^{25}=+10^{\circ}\left(\mathrm{CHCl}_{3}\right) ; \quad \mathrm{HPLC}$ analysis Chiracel OD-H column, 97:3 hexanes to isopropanol $0.5 \mathrm{ml} / \mathrm{min}$. Minor enantiomer: 19.1 minutes, major enantiomer: 33.7 minutes; ${ }^{1} \mathrm{H}$ NMR $\left(300 \mathrm{MHz}, \mathrm{CDCl}_{3}\right) \square 7.79$ (d, $1 \mathrm{H}, J=7.7 \mathrm{~Hz}), 7.53(\mathrm{dd}, 1 \mathrm{H}, J=7.3,0 \mathrm{~Hz}), 7.38(\mathrm{~d}, 1 \mathrm{H}, J=7.7 \mathrm{~Hz}), 7.2(\mathrm{dd}, 1 \mathrm{H}, J=$ 7.3, $0 \mathrm{~Hz}), 3.57(\mathrm{~s}, 3 \mathrm{H}), 3.07(\mathrm{~d}, 1 \mathrm{H}, J=16.9 \mathrm{~Hz}), 2.97(\mathrm{~d}, 1 \mathrm{H}, J=16.5 \mathrm{~Hz}), 1.94(\mathrm{q}, 2 \mathrm{H}$, $J=7.3 \mathrm{~Hz}), 0.88(\mathrm{t}, 3 \mathrm{H}, J=7.3 \mathrm{~Hz}) ;{ }^{13} \mathrm{C} \mathrm{NMR}\left(100 \mathrm{MHz}, \mathrm{CDCl}_{3}\right) \square 203.9,170.5,152.2$, 135.8, 131.5, 126.8, 124.9, 124.2, 63.4, 52.0, 42.6, 34.3, 9.0; IR (NaCl, neat) 2964, 1730, $1701,1594,1429 \mathrm{~cm}^{-1}$; HRMS (EI+) calcd for $\mathrm{C}_{13} \mathrm{H}_{14} \mathrm{O}_{3} \mathrm{~S}, 250.0664$. Found 250.0653.

(R)-(2-Methyl-1-oxo-indan-2-yl)-acetic acid ethyl ester (12): According to the general

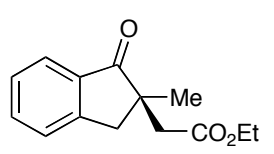
procedure, aldehyde $11(20.0 \mathrm{mg}, 0.085 \mathrm{mmol})$ in toluene $(1 \mathrm{~mL})$ was added to catalyst $\mathbf{5 c}(8.0 \mathrm{mg}, 0.017 \mathrm{mmol})$ followed by addition of triethylamine $(0.025 \mathrm{~mL}, 0.17 \mathrm{mmol})$ and allowed to stir at ambient temperature for 24 hours. Flash column chromatography (6:1 hexanes to ethyl acetate) provided $19.0 \mathrm{mg}(95 \%)$ of $\mathbf{1 2}$ as a clear oil: $[\square]_{\mathrm{D}}{ }^{25}=+22^{\circ}(\mathrm{EtOH})$; HPLC analysis - Chiracel OD-H column, 97:3 hexanes to isopropanol $1.0 \mathrm{ml} / \mathrm{min}$. Minor enantiomer: 7.2 minutes, Major enantiomer: 11.1 minutes. Spectral data matched literature description. ${ }^{2}$

(R)-3-(2-Oxo-propyl)-3-phenyl-chroman-4-one (14) According to the general<smiles>CC(=O)CC1(O)COc2ccccc2C1=O</smiles>
procedure, aldehyde $13(20.0 \mathrm{mg}, 0.071 \mathrm{mmol})$ in toluene $(1 \mathrm{~mL})$ was added to catalyst $\mathbf{5 c}(3.5 \mathrm{mg}, 0.007 \mathrm{mmol})$ followed by addition of triethylamine $(0.010 \mathrm{~mL}, 0.070 \mathrm{mmol})$ and allowed to stir at ambient temperature for 12 hours. At this time, another aliquot of catalyst (3.5 $\mathrm{mg}, 0.007 \mathrm{mmol})$ was added with another 2 eq triethylamine $(0.010 \mathrm{~mL}, 0.070 \mathrm{mmol})$ and allowed to stir for another 12 hours at $25{ }^{\circ} \mathrm{C}$. Flash column chromatography $(6: 1$ hexanes to ethyl acetate) provided $11.0 \mathrm{mg}(55 \%)$ of $\mathbf{1 4}$ as a colorless oil: $\mathrm{Rf}(2: 1$ hexanes to ethyl acetate $)=0.34 ;[\square]_{\mathrm{D}}{ }^{25}=-88^{\circ}\left(\mathrm{CH}_{2} \mathrm{Cl}_{2}\right)$; HPLC analysis - Chiracel OD-H column, 95:5 hexanes to isopropanol $1.0 \mathrm{ml} / \mathrm{min}$. Minor enantiomer: 14.3 minutes, major enantiomer: 19.5 minutes; ${ }^{1} \mathrm{H}$ NMR $\left(300 \mathrm{MHz}, \mathrm{CDCl}_{3}\right) \square 7.90(\mathrm{dd}, 1 \mathrm{H}, J=7.7,1.3 \mathrm{~Hz})$, $7.41(\mathrm{~d}, 2 \mathrm{H}, J=7.9 \mathrm{~Hz}), 7.36(\mathrm{ddd}, 1 \mathrm{H}, J=8.7,1.5,0 \mathrm{~Hz}), 7.29(\mathrm{dd}, 2 \mathrm{H}, J=7.3,0 \mathrm{~Hz})$, $7.21(\mathrm{dd}, 1 \mathrm{H}, J=7.2,0 \mathrm{~Hz}), 6.94(\mathrm{dd}, 1 \mathrm{H}, J=7.2,0 \mathrm{~Hz}), 6.83(\mathrm{~d}, 1 \mathrm{H}, J=8.3 \mathrm{~Hz}), 5.10$ $(\mathrm{d}, 1 \mathrm{H}, J=12.1 \mathrm{~Hz}), 4.99(\mathrm{~d}, 1 \mathrm{H}, J=12.0 \mathrm{~Hz}), 3.18(\mathrm{~d}, 1 \mathrm{H}, J=17.5 \mathrm{~Hz}), 2.99(\mathrm{~d}, 1 \mathrm{H}, \mathrm{J}=$ $17.5 \mathrm{~Hz}), 2.03$ (s, 3H); ${ }^{13} \mathrm{C}$ NMR (100 MHz, $\left.\mathrm{CDCl}_{3}\right) \square 206.1,193.2,161.1,136.7,136.1$, 129.1, 128.1, 128.0, 126.9, 121.5, 120.3, 117.8, 71.5, 51.7, 49.1, 31.3; IR (NaCl, neat) 2923, 1717, 1684, 1606, 1408, $1458 \mathrm{~cm}^{-1}$; HRMS (FAB+) calcd for M+H, 281.1178. Found 281.1175.

\footnotetext{
${ }^{2}$ Iwaya, K.; Tamura, T.; Nakamura, M.; Hasegawa, E. Tetrahedron Lett. 2003, 44, 9317.
} 
(S)-2-Methyl-2-(2-oxo-2-phenyl-ethyl)-cyclopentanone (16): According to the general procedure, catalyst $\mathbf{5 c}(5.5 \mathrm{mg}, 0.012 \mathrm{mmol})$ in toluene $(1 \mathrm{~mL})$ was treated with KHMDS $(0.5 \mathrm{M}$ in toluene) $(0.024 \mathrm{~mL}, 0.012 \mathrm{mmol})$ and allowed to stir at ambient temperature for 5 minutes. Aldehyde $\boldsymbol{E}-\mathbf{1 5}(13.0 \mathrm{mg}, 0.060 \mathrm{mmol})$ in toluene $(1 \mathrm{~mL})$ was added via syringe and the reaction was stirred for $24 \mathrm{~h}$ at $25{ }^{\circ} \mathrm{C}$. Flash column chromatography (6:1 hexanes to ethyl acetate) provided $11.0 \mathrm{mg}(85 \%)$ of $\mathbf{1 6}$ as a white solid: $\operatorname{Rf}(3: 1$ hexanes to ethyl acetate $)=0.35$; $[\square]_{\mathrm{D}}{ }^{25}=-4^{\mathrm{O}}\left(\mathrm{CH}_{2} \mathrm{Cl}_{2}\right)$; HPLC analysis - Chiracel OD-H column, 97:3 hexanes to isopropanol $1.0 \mathrm{ml} / \mathrm{min}$. Minor enantiomer: 9.8 minutes, Major enantiomer: 11.2 minutes; ${ }^{1} \mathrm{H}$ NMR $\left(300 \mathrm{MHz}, \mathrm{CDCl}_{3}\right) \square 7.92(\mathrm{~d}, 2 \mathrm{H}, J=7.3 \mathrm{~Hz}), 7.55$ (dd, $1 \mathrm{H}, J=6.2,0$ $\mathrm{Hz}), 7.44(\mathrm{dd}, 2 \mathrm{H}, J=7.7,0 \mathrm{~Hz}), 3.36(\mathrm{~d}, 1 \mathrm{H}, J=20.0 \mathrm{~Hz}), 3.29(\mathrm{~d}, 1 \mathrm{H}, J=20.0 \mathrm{~Hz})$, 2.68 (ddd, 1H, $J=18.7,9.2,1.5 \mathrm{~Hz}$ ), 2.39 (dddd, 1H, J = 18.7, 6.6, 2.2, $2.2 \mathrm{~Hz}$ ), 1.77 $2.22(\mathrm{~m}, 4 \mathrm{H}), 1.11(\mathrm{~s}, 3 \mathrm{H}) ;{ }^{13} \mathrm{C} \mathrm{NMR}\left(100 \mathrm{MHz} \mathrm{CDCl}_{3}\right) \square 223.2,198.0,136.9,133.4$, 128.8, 128.1, 46.7, 46.1, 37.4, 35.1, 23.4, 19.2; IR (NaCl, neat) 2955, 1727, 1679, 1398, $1356 \mathrm{~cm}^{-1}$; HRMS (EI+) calcd for $\mathrm{C}_{14} \mathrm{H}_{16} \mathrm{O}_{2}, 216.1150$. Found 216.1147.

(S)-2-Methyl-2-(2-oxo-2-pyridin-4-yl-ethyl)-cyclopentanone (18): According to the

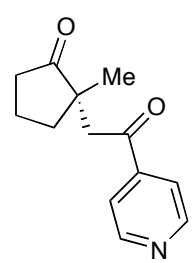
general procedure, catalyst $\mathbf{5 c}(8.5 \mathrm{mg}, 0.018 \mathrm{mmol})$ in toluene $(1 \mathrm{~mL})$ was treated with KHMDS $(0.5 \mathrm{M}$ in toluene) $(0.036 \mathrm{~mL}, 0.018 \mathrm{mmol})$ and allowed to stir at ambient temperature for 5 minutes. Aldehyde 17 (20.0 mg, 0.092 $\mathrm{mmol})$ in toluene $(1 \mathrm{~mL})$ was added via syringe and the reaction was stirred for $24 \mathrm{~h}$ at $25^{\circ} \mathrm{C}$. Flash column chromatography (1:1 hexanes to ethyl acetate) provided $17.0 \mathrm{mg}(85 \%)$ of $\mathbf{1 8}$ as a colorless oil: $\mathrm{Rf}$ (ethyl acetate) $=0.28$; $[\square]_{\mathrm{D}}^{25}=-0.8^{\mathrm{o}}\left(\mathrm{CH}_{2} \mathrm{Cl}_{2}\right)$; HPLC analysis - Chiracel OB-H column, 90:10 hexanes to isopropanol $1.0 \mathrm{ml} / \mathrm{min}$. Minor enantiomer: 25.0 minutes, major enantiomer: 28.1 minutes; ${ }^{1} \mathrm{H}$ NMR $\left(300 \mathrm{MHz}, \mathrm{CDCl}_{3}\right) \square 8.80$ (bs, 2H), 7.69 (bs, 2H), 3.32 (d, 1H, J = $18.7 \mathrm{~Hz}$ ), $3.27(\mathrm{~d}, 1 \mathrm{H}, J=19.0 \mathrm{~Hz}), 2.54-2.68(\mathrm{~m}, 1 \mathrm{H}), 2.40$ (dddd, $1 \mathrm{H}, J=18.3,7.7$, 0.0, $0.0 \mathrm{~Hz}), 1.77-2.20(\mathrm{~m}, 4 \mathrm{H}), 1.11(\mathrm{~s}, 3 \mathrm{H}) ;{ }^{13} \mathrm{C} \mathrm{NMR}\left(100 \mathrm{MHz}, \mathrm{CDCl}_{3}\right) \square 222.6$, 197.5, 151.1, 142.7, 121.1, 46.7, 46.1, 37.3, 34.9, 23.2, 19.1; IR (NaCl, neat) 2964, 1736, 1697, 1408, 1356, $1222 \mathrm{~cm}^{-1}$; HRMS (Fab+) calcd for M+H, 218.1181. Found 218.1172.

(S)-2-Methyl-2-[2-(4-nitro-phenyl)-2-oxo-ethyl]-cyclopentanone (20): According to the general procedure, catalyst $5 \mathbf{c}(7.0 \mathrm{mg}, 0.015 \mathrm{mmol})$ in toluene $(1 \mathrm{~mL})$

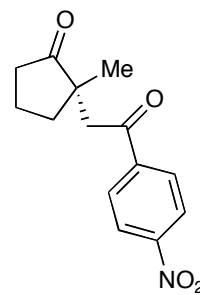
was treated with KHMDS $(0.5 \mathrm{M}$ in toluene) $(0.030 \mathrm{~mL}, 0.015 \mathrm{mmol})$ and allowed to stir at ambient temperature for 5 minutes. Aldehyde 19 (20.0 mg, $0.077 \mathrm{mmol})$ in toluene $(1 \mathrm{~mL})$ was added via syringe and the reaction was stirred for $24 \mathrm{~h}$ at $25^{\circ} \mathrm{C}$. Flash column chromatography (4:1 hexanes to ethyl acetate) provided $18.0 \mathrm{mg}(90 \%)$ of $\mathbf{2 0}$ as a yellow solid: $\mathrm{Rf}$ (2:1 hexanes to $\mathrm{NO}_{2}$ ethyl acetate $)=0.40 ;[\square]_{\mathrm{D}}{ }^{25}=-17^{\circ}\left(\mathrm{CHCl}_{3}\right) ; \mathrm{HPLC}$ analysis - Chiracel OD-H column, 97:3 hexanes to isopropanol $1.0 \mathrm{ml} / \mathrm{min}$. Minor enantiomer: 36.3 minutes, major enantiomer: 44.8 minutes; ${ }^{1} \mathrm{H}$ NMR $\left(300 \mathrm{MHz}, \mathrm{CDCl}_{3}\right) \square 8.27(\mathrm{~d}, 2 \mathrm{H}, J=9.0 \mathrm{~Hz}), 8.05$ $(\mathrm{d}, 2 \mathrm{H}, J=8.4 \mathrm{~Hz}), 3.36(\mathrm{~d}, 1 \mathrm{H}, J=18.3 \mathrm{~Hz}), 3.30(\mathrm{~d}, 1 \mathrm{H}, J=18.3 \mathrm{~Hz}), 2.61$ (ddd, $1 \mathrm{H}, J$ $=19.0,10.9,1.5 \mathrm{~Hz}), 2.40$ (dddd $J=18.8,8.3,0,0 \mathrm{~Hz}), 2.02-2.19(\mathrm{~m}, 2 \mathrm{H}),, 1.85-$ $1.98(\mathrm{~m}, 1 \mathrm{H}), 1.77-1.84(\mathrm{~m}, 1 \mathrm{H}), 1.11(\mathrm{~s}, 3 \mathrm{H}) ;{ }^{13} \mathrm{C} \mathrm{NMR}\left(100 \mathrm{MHz}, \mathrm{CDCl}_{3}\right) \square 222.6$, 
196.1, 150.6, 141.3, 129.2, 124.1, 47.0, 46.2, 37.3, 34.9, 23.3, 19.1; IR (NaCl, neat) 3107, 2963, 1736, 1690, 1524, $1345 \mathrm{~cm}^{-1}$; HRMS (Fab+) calcd for $\mathrm{M}+\mathrm{H}, 262.1079$. Found 262.1068 .

(S)-2-Methyl-2(2-oxo-propyl)-cyclopentanone (22): According to the general procedure, catalyst $\mathbf{5 c}(9.5 \mathrm{mg}, 0.020 \mathrm{mmol})$ in toluene $(1 \mathrm{~mL})$ was treated with KHMDS $(0.5 \mathrm{M}$ in toluene) $(0.040 \mathrm{~mL}, 0.020 \mathrm{mmol})$ and allowed to stir at ambient temperature for 5 minutes. Aldehyde 21 (16.0 $\mathrm{mg}, 0.10 \mathrm{mmol})$ in toluene $(1 \mathrm{~mL})$ was added via syringe and the reaction was stirred for $24 \mathrm{~h}$ at $25{ }^{\circ} \mathrm{C}$. Flash column chromatography $(6: 1$ hexanes to ethyl acetate) provided $13.0 \mathrm{mg}$ $(82 \%)$ of 22 as a colorless oil: $[\square]_{\mathrm{D}}^{25}=+11^{\circ}(\mathrm{EtOH})$; Gas chromatography analysisChiraldex B-DM column, gas flow $3 \mathrm{ml} / \mathrm{min}$ with constant $110{ }^{\circ} \mathrm{C}$ oven temperature. Minor enantiomer: 12.9 min, major enantiomer: 14.5 minutes. Spectral data matched literature description. ${ }^{3}$

(S)-2-Methyl-2-(2-oxo-4-phenyl-butyl)-cyclopentanone (24): According to the general

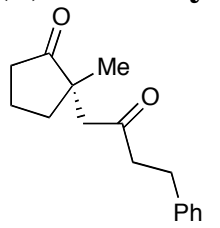
procedure, catalyst $5 \mathbf{c}(6.0 \mathrm{mg}, 0.013 \mathrm{mmol})$ in toluene $(1 \mathrm{~mL})$ was treated with KHMDS $(0.5 \mathrm{M}$ in toluene) $(0.026 \mathrm{~mL}, 0.013 \mathrm{mmol})$ and allowed to stir at ambient temperature for 5 minutes. Aldehyde 23 (16.0 mg, 0.066 $\mathrm{mmol})$ in toluene $(1 \mathrm{~mL})$ was added via syringe and the reaction was stirred for $24 \mathrm{~h}$ at $25^{\circ} \mathrm{C}$. Flash column chromatography (4:1 hexanes to ethyl acetate) provided $10.0 \mathrm{mg}(63 \%)$ of the $\mathbf{2 4}$ as a colorless oil: $\mathrm{Rf}$ (3:1 hexanes to ethyl acetate $)=0.38 ;[\square]_{\mathrm{D}}{ }^{25}=+22^{\circ}\left(\mathrm{CH}_{2} \mathrm{Cl}_{2}\right)$; HPLC analysis - Chiraldex B-DM capillary gas chromatography column, $150{ }^{\circ} \mathrm{C}$ constant oven temperature, constant gas flow $3.0 \mathrm{ml} /$ min. Minor enantiomer 119 minutes: major enantiomer: 125 minutes; ${ }^{1} \mathrm{H}$ NMR (300 $\left.\mathrm{MHz}, \mathrm{CDCl}_{3}\right) \square 7.27(\mathrm{dd}, 2 \mathrm{H}, J=6.6,0 \mathrm{~Hz}), 7.19(\mathrm{~d}, 1 \mathrm{H}, J=7.4 \mathrm{~Hz}), 7.19$ (d, 2H, $J=6.8$ $\mathrm{Hz}), 2.76-2.80(\mathrm{~m}, 3 \mathrm{H}), 2.58-2.69(\mathrm{~m}, 3 \mathrm{H}), 2.51(\mathrm{ddd}, 1 \mathrm{H}, J=18.3,9.5,1.8 \mathrm{~Hz}), 2.33$ (dddd, $1 \mathrm{H}, J=18.7,7.3,2.2,2.2 \mathrm{~Hz}), 1.65-2.10(\mathrm{~m}, 4 \mathrm{H}), 0.97(\mathrm{~s}, 3 \mathrm{H}) ;{ }^{13} \mathrm{C}$ NMR $(100$ $\left.\mathrm{MHz}_{\mathrm{CDCl}}\right) \square$ 223.0, 208.0, 141.0, 128.7, 128.5, 126.3, 50.7, 46.0, 44.6, 37.4, 35.0, 29.8, 23.1, 19.1; IR (NaCl, neat) 2961, 1736, 1713, 1454, $1072 \mathrm{~cm}^{-1}$; HRMS (EI+) calcd for $\mathrm{C}_{16} \mathrm{H}_{20} \mathrm{O}_{2}, 244.1463$. Found 244.1474.

(S)-2-Butyl-2-(2-oxo-2-phenyl-ethyl)-cyclopentanone (26): According to the general procedure, catalyst 5c $(5.0 \mathrm{mg}, 0.011 \mathrm{mmol})$ in toluene $(1 \mathrm{~mL})$ was treated
with KHMDS $(0.5 \mathrm{M}$ in toluene $)(0.022 \mathrm{~mL}, 0.011 \mathrm{mmol})$ and allowed to stir
at ambient temperature for 5 minutes. Aldehyde $25(14.0 \mathrm{mg}, 0.055 \mathrm{mmol})$
in toluene $(1 \mathrm{~mL})$ was added via syringe and the reaction was stirred for $24 \mathrm{~h}$ provided $10.0 \mathrm{mg}(71 \%)$ of $\mathbf{2 6}$ as a colorless oil: $\operatorname{Rf}(4: 1$ hexanes to ethyl acetate $)=0.28$; $[\square]_{\mathrm{D}}^{25}=-30^{\circ}\left(\mathrm{CH}_{3} \mathrm{CN}\right)$; HPLC analysis - Chiracel OD-H column, 97:3 hexanes to isopropanol $1.0 \mathrm{ml} / \mathrm{min}$. Major enantiomer: 7.4 minutes, minor enantiomer: 9.2 minutes; ${ }^{1} \mathrm{H}$ NMR $\left(300 \mathrm{MHz}, \mathrm{CDCl}_{3}\right) \square 7.92(\mathrm{~d}, 2 \mathrm{H}, J=7.3 \mathrm{~Hz}), 7.55 \mathrm{dd},(1 \mathrm{H}, J=7.3,0 \mathrm{~Hz}), 7.44$

\footnotetext{
${ }^{3}$ Thominiaux, C.; Roussé, S.; Desmaële, D.; d'Angelo, J.; Riche, C. Tetrahedron: Asymmetry. 1999, 10, 2015 .
} 
(dd, 2H, $J=7.7,0 \mathrm{~Hz}), 3.37(\mathrm{~d}, 1 \mathrm{H}, J=18.3 \mathrm{~Hz}), 3.30(\mathrm{~d}, 1 \mathrm{H}, J=18.0 \mathrm{~Hz}), 2.70$ (ddd, $1 \mathrm{H}, J=19.0,9.5,1.5 \mathrm{~Hz}), 2.35(\mathrm{dddd}, 1 \mathrm{H}, J=18.7,7.3,2.2,2.2 \mathrm{~Hz}), 1.99-2.14(\mathrm{~m}$, $2 \mathrm{H}), 1.79-1.98(\mathrm{~m}, 2 \mathrm{H}), 1.14-1.54(\mathrm{~m}, 6 \mathrm{H}) 0.92(\mathrm{t}, 3 \mathrm{H}, J=6.6 \mathrm{~Hz}) ;{ }^{13} \mathrm{C}$ NMR $(100$ $\left.\mathrm{MHz}_{\mathrm{CDCl}}\right) \square 223.4,198.2,137.0,133.4,128.7,128.2,49.4,45.3$, 37.8, 36.4, 33.7, 26.7, 23.5, 19.1, 14.2; IR (NaCl, neat) 2957, 2929, 2858, 1734, 1685, $1158 \mathrm{~cm}^{-1}$; HRMS (EI+) calcd for $\mathrm{C}_{17} \mathrm{H}_{22} \mathrm{O}_{2}, 258.1620$, Found 258.1611 .

(S)-1-Methoxycarbonylmethyl-2-oxo-cyclopentanecarboxylic acid methyl ester (30):

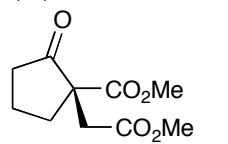
According to the general procedure, catalyst $5 \mathbf{c}(4.0 \mathrm{mg}, 0.013 \mathrm{mmol})$ in toluene $(1 \mathrm{~mL})$ was treated with KHMDS $(0.5 \mathrm{M}$ in toluene) $(0.026 \mathrm{~mL}$, $0.013 \mathrm{mmol}$ ) and allowed to stir at ambient temperature for 5 minutes. Aldehyde $29(14.0 \mathrm{mg}, 0.065 \mathrm{mmol})$ in toluene $(1 \mathrm{~mL})$ was added via syringe and the reaction was stirred for $12 \mathrm{~h}$ at $25^{\circ} \mathrm{C}$. Flash column chromatography $(4: 1$ hexanes to ethyl acetate) provided $12.0 \mathrm{mg}(86 \%)$ of $\mathbf{3 0}$ as a colorless oil: $[\square]_{\mathrm{D}}{ }^{25}=-20^{\circ}$ $(\mathrm{EtOH})$; Gas chromatography analysis - Chiraldex B-PH column with constant $110{ }^{\circ} \mathrm{C}$ oven temperature and constant flow of $1 \mathrm{ml} / \mathrm{min}$. Major enantiomer: 169.3 minutes, minor enantiomer: 170.6 min. Spectral data matched literature description. ${ }^{4}$

\footnotetext{
${ }^{4}$ Matsui, J.; Bando, M.; Kido, M.; Takeuchi, Y.; Mori, K. Eur. J. Org. Chem. 1999, 2183.
} 

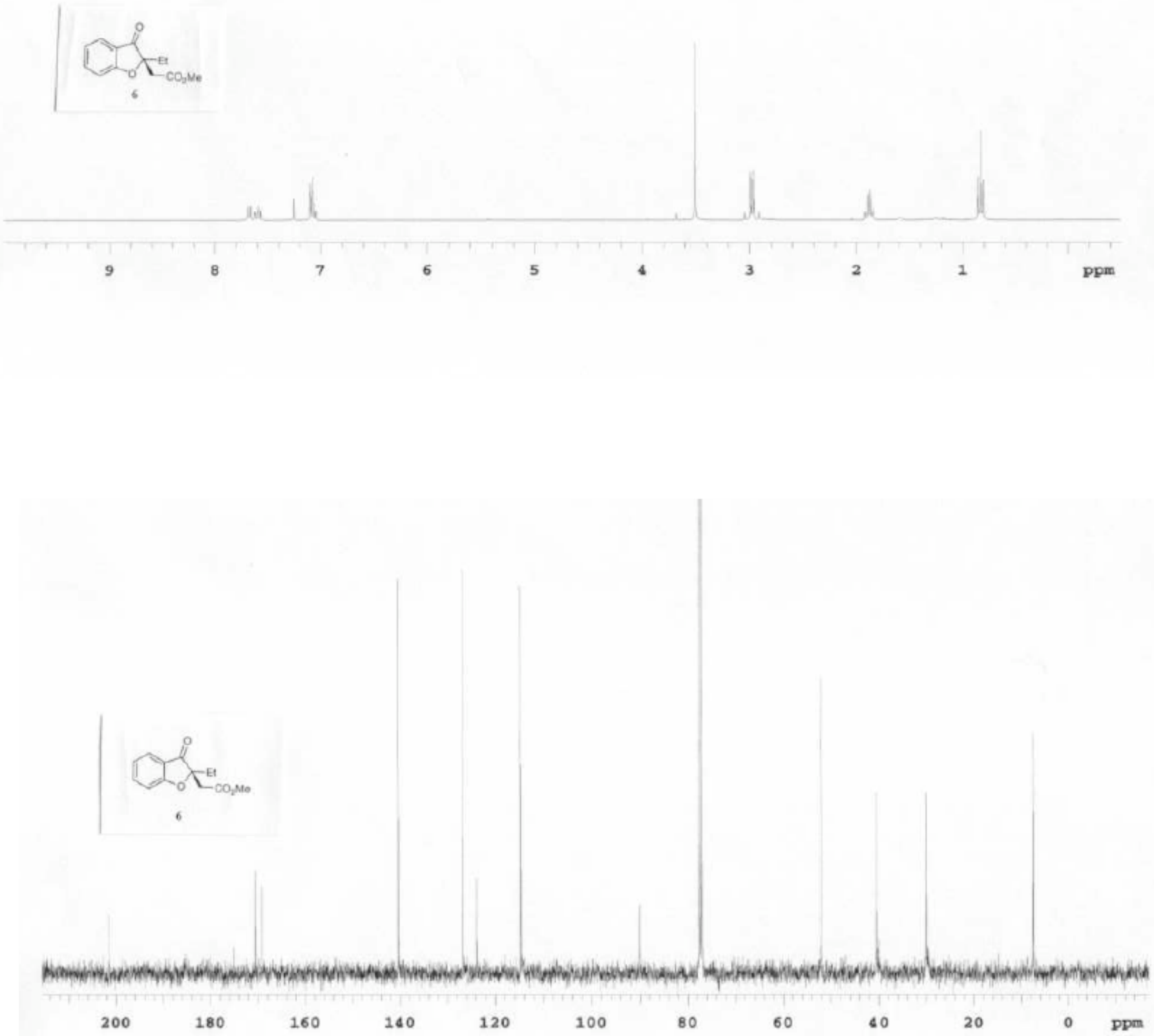


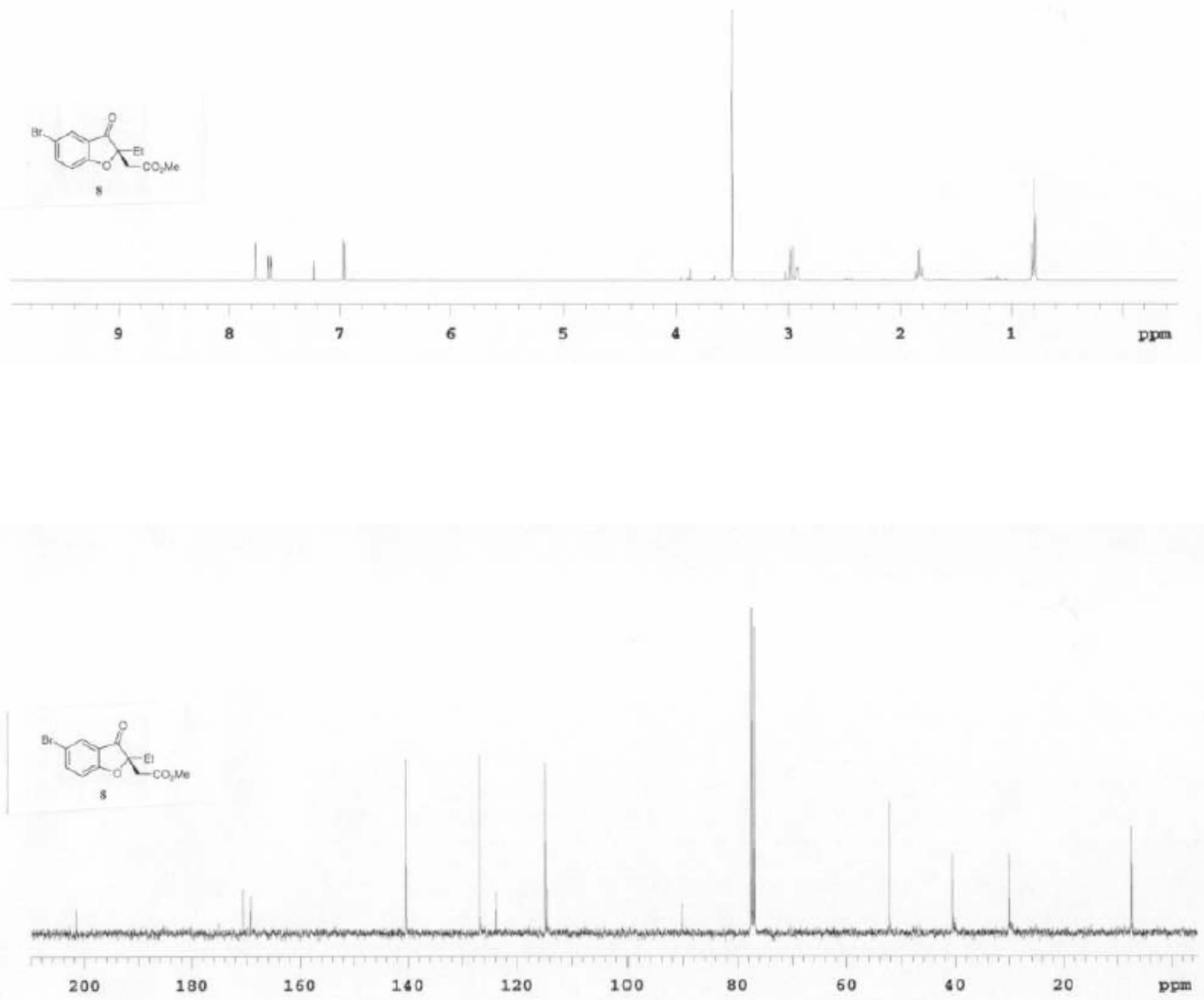



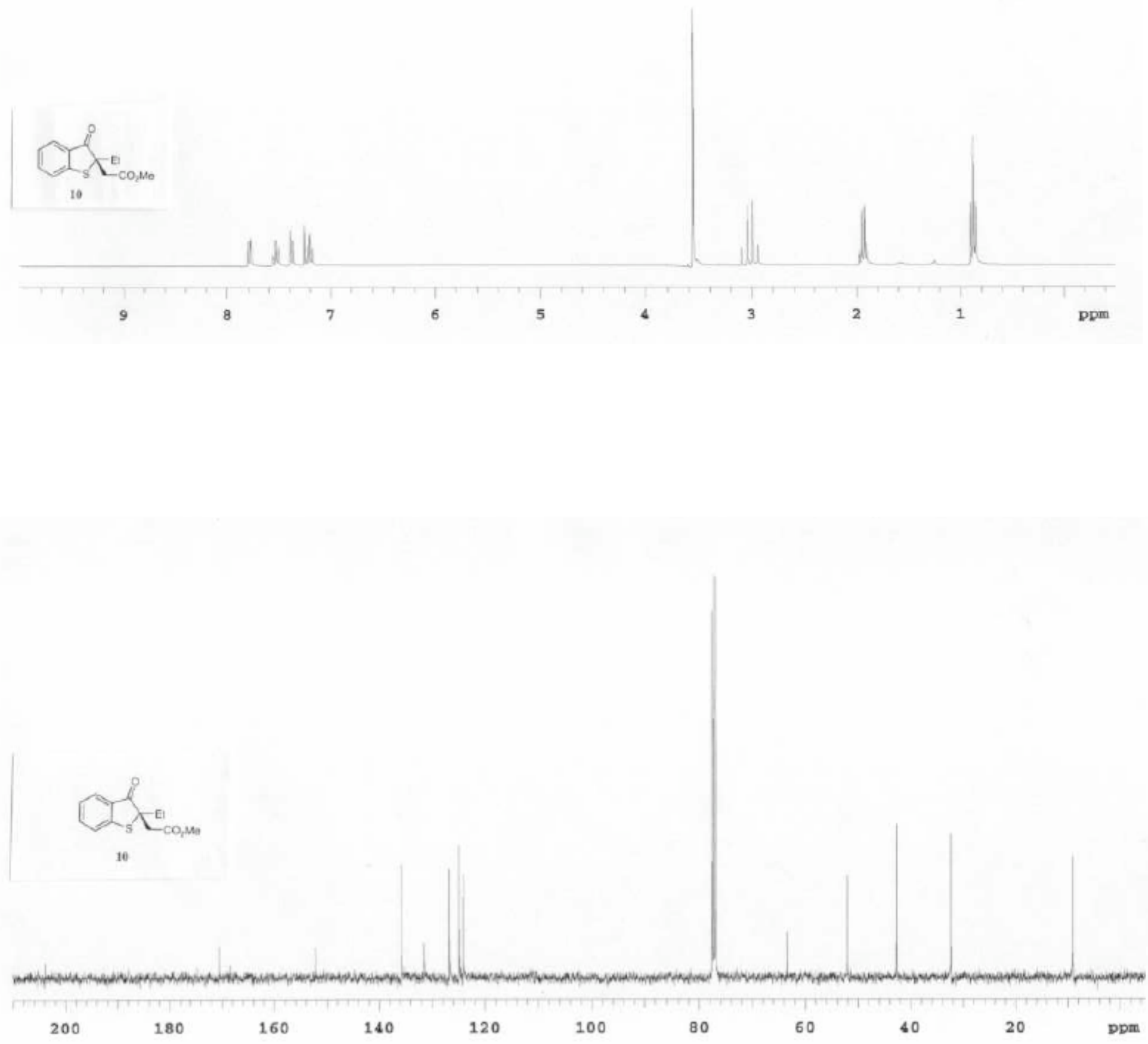

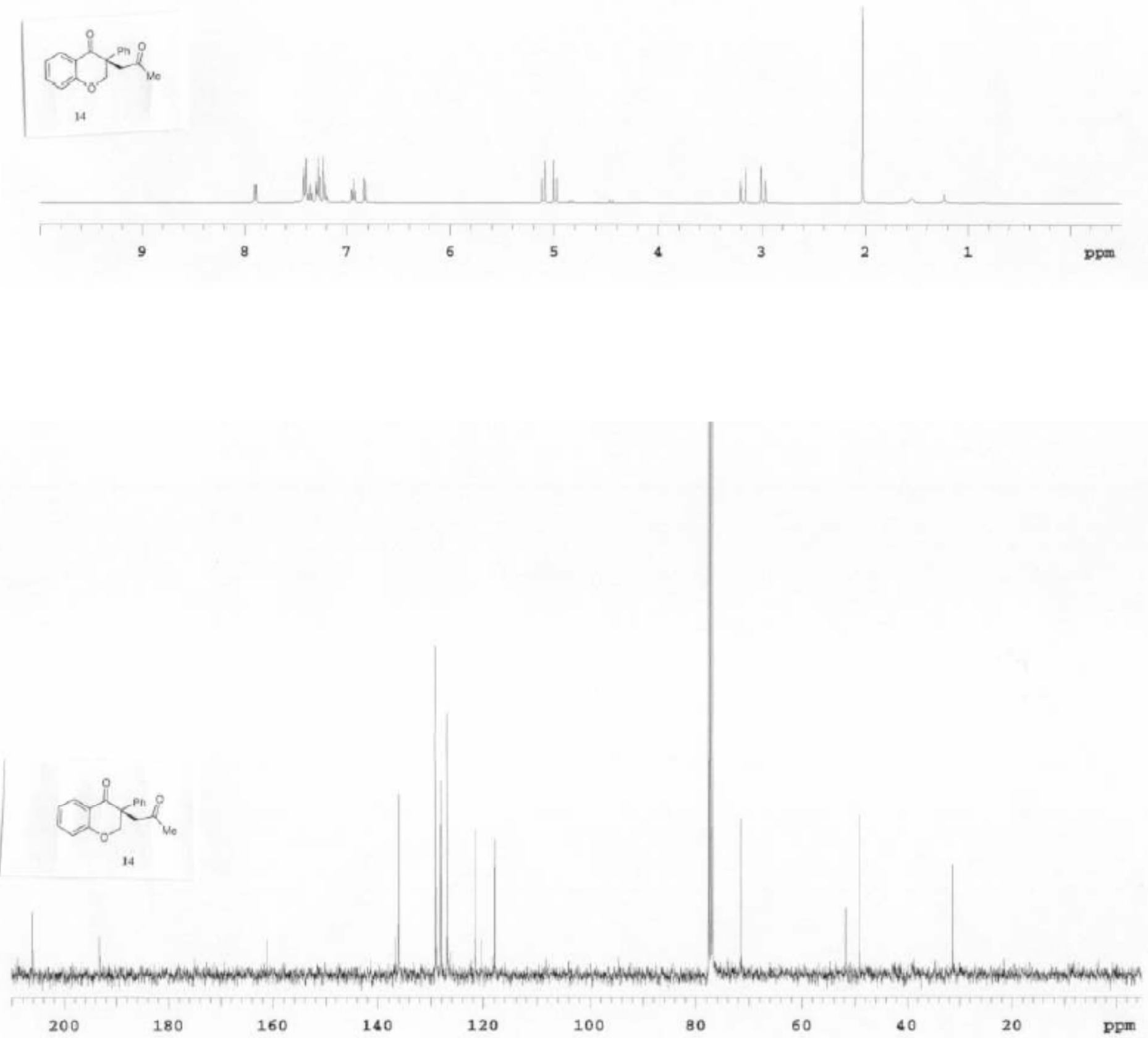


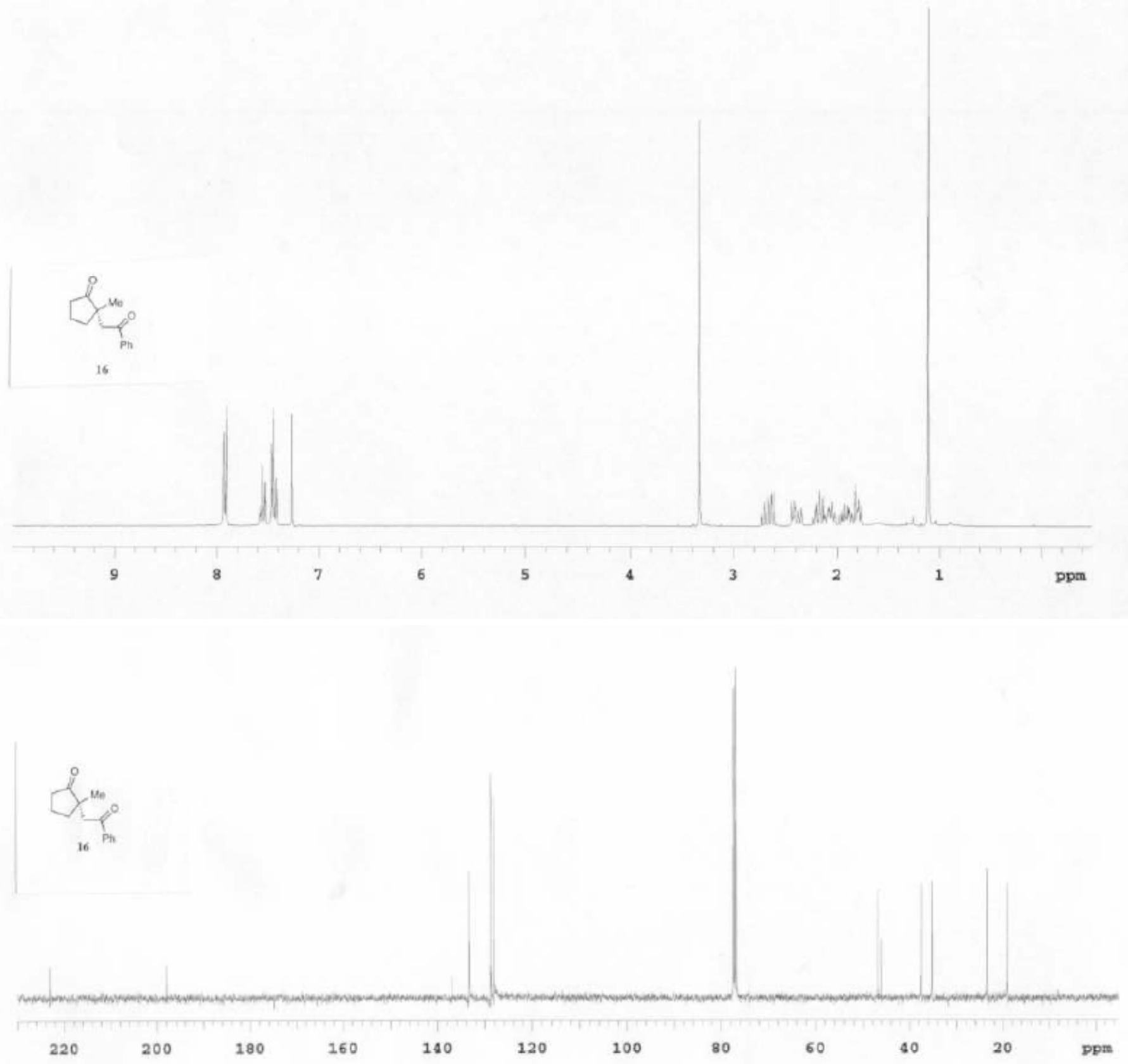



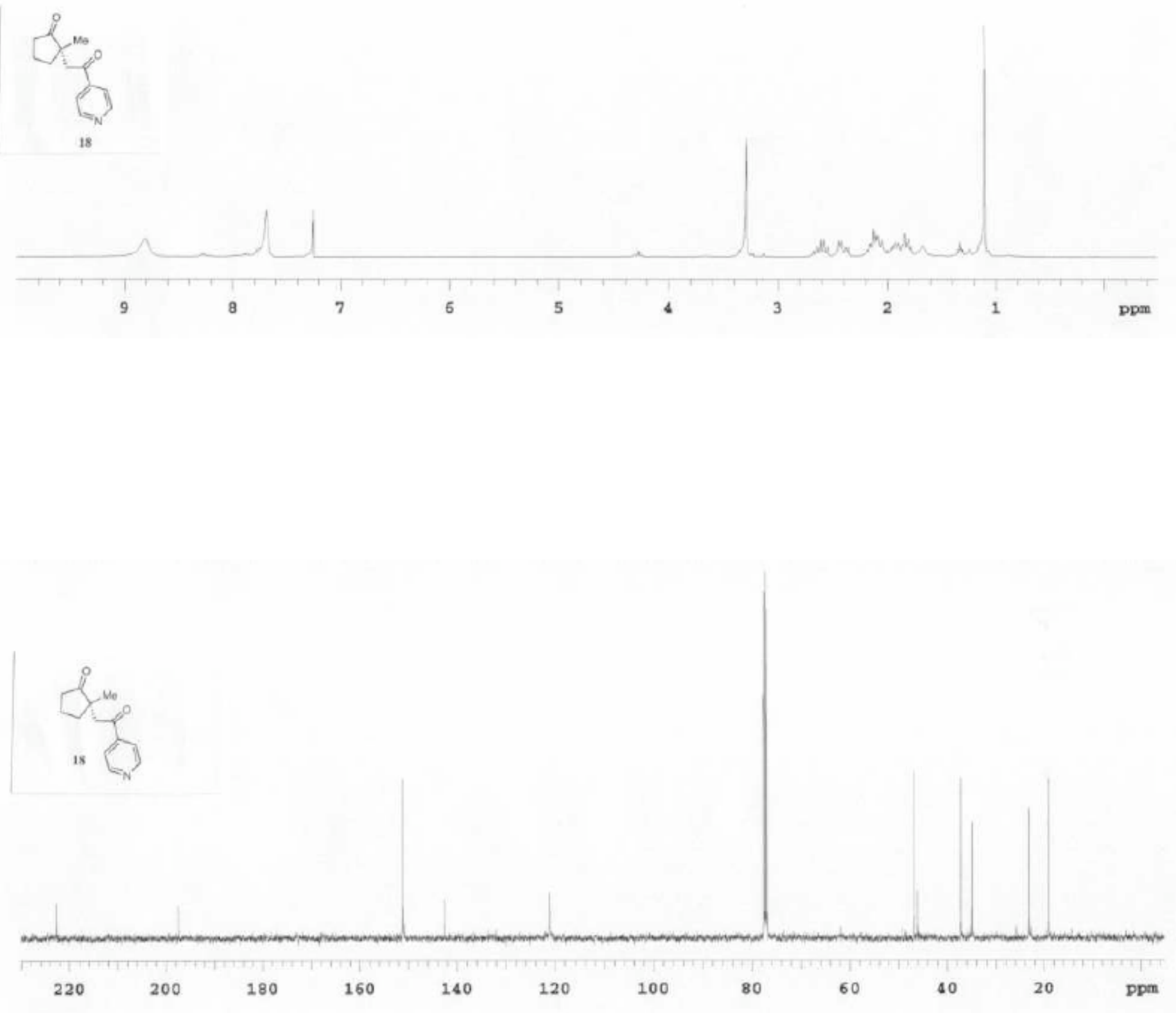

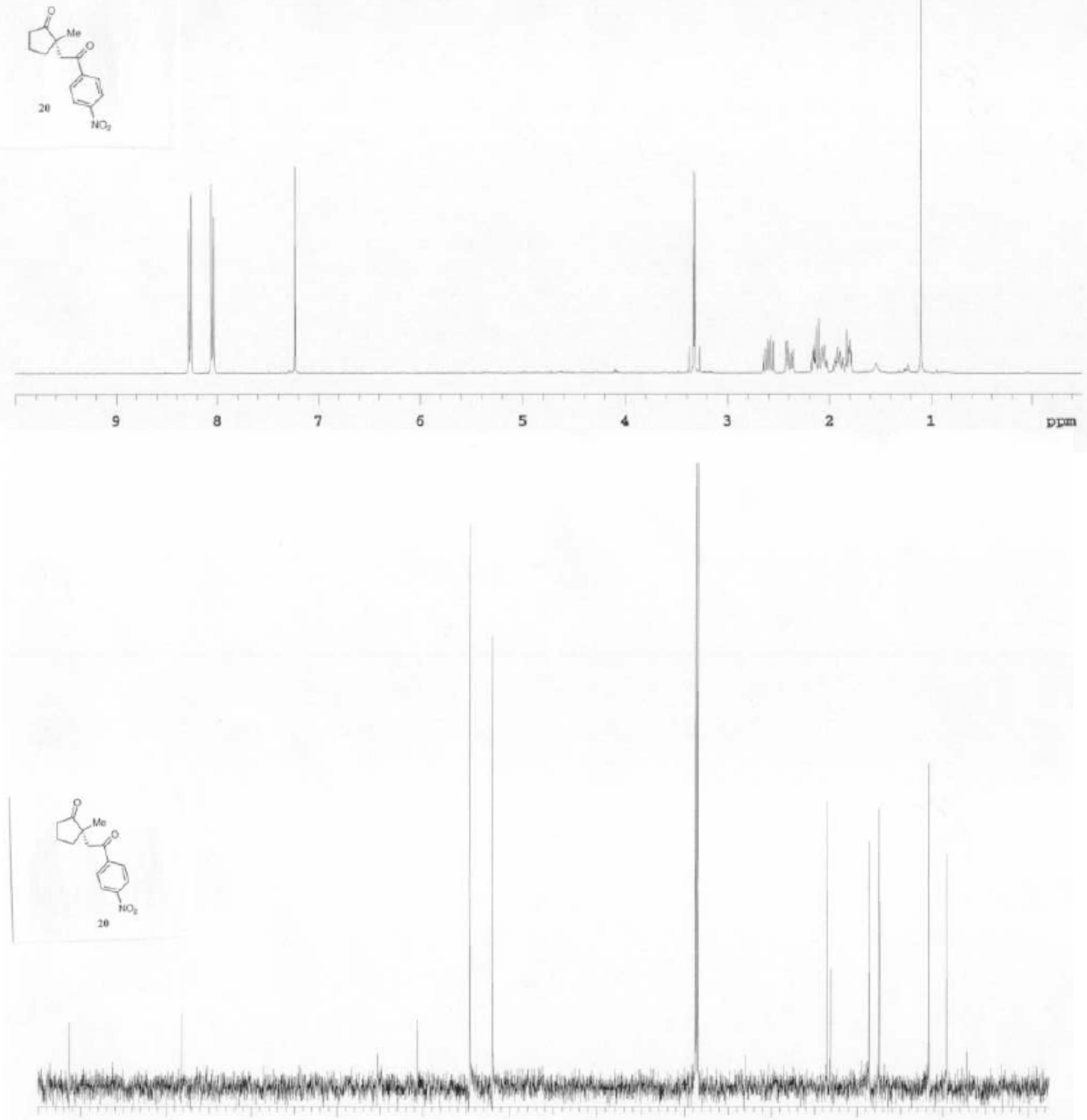

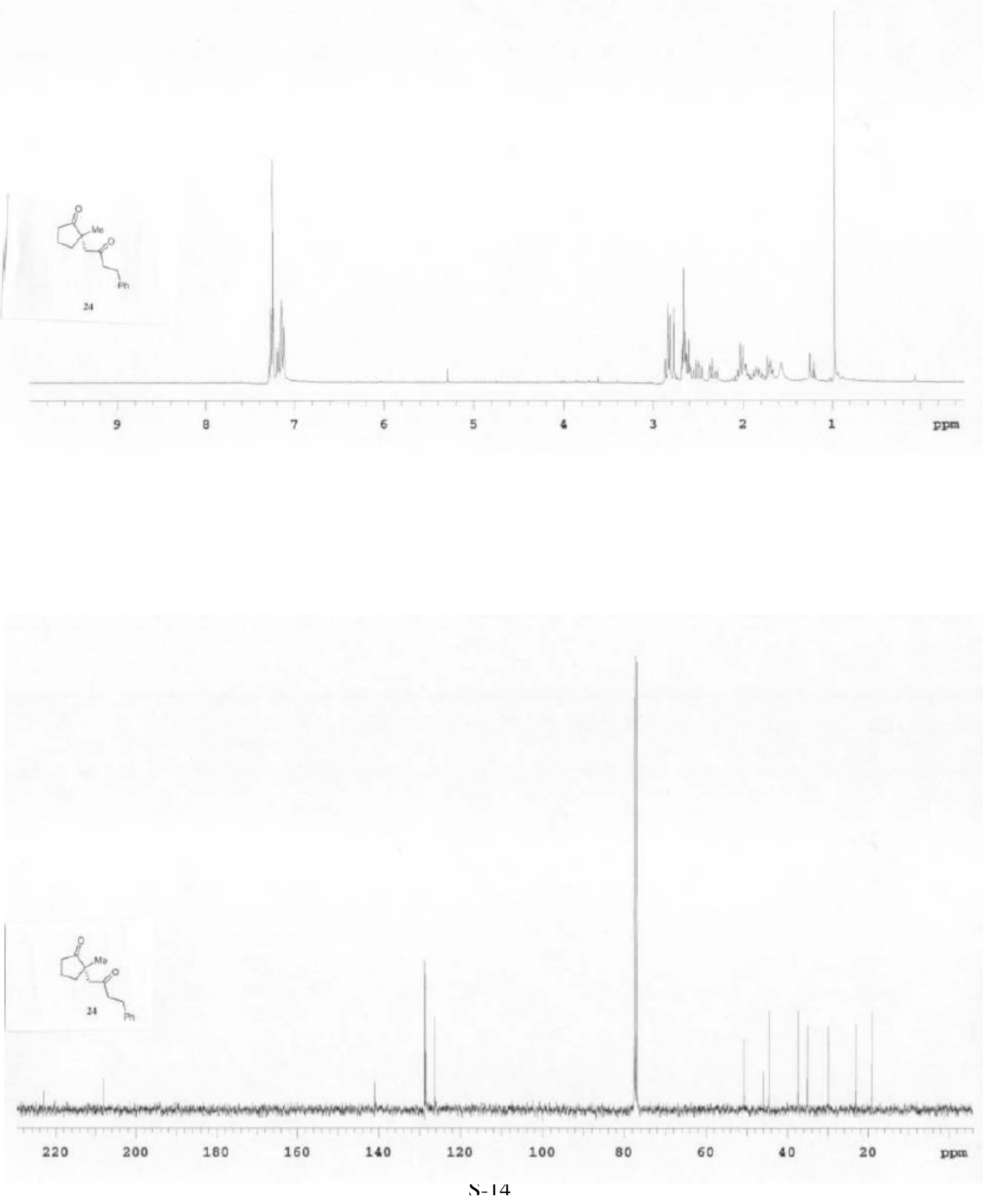

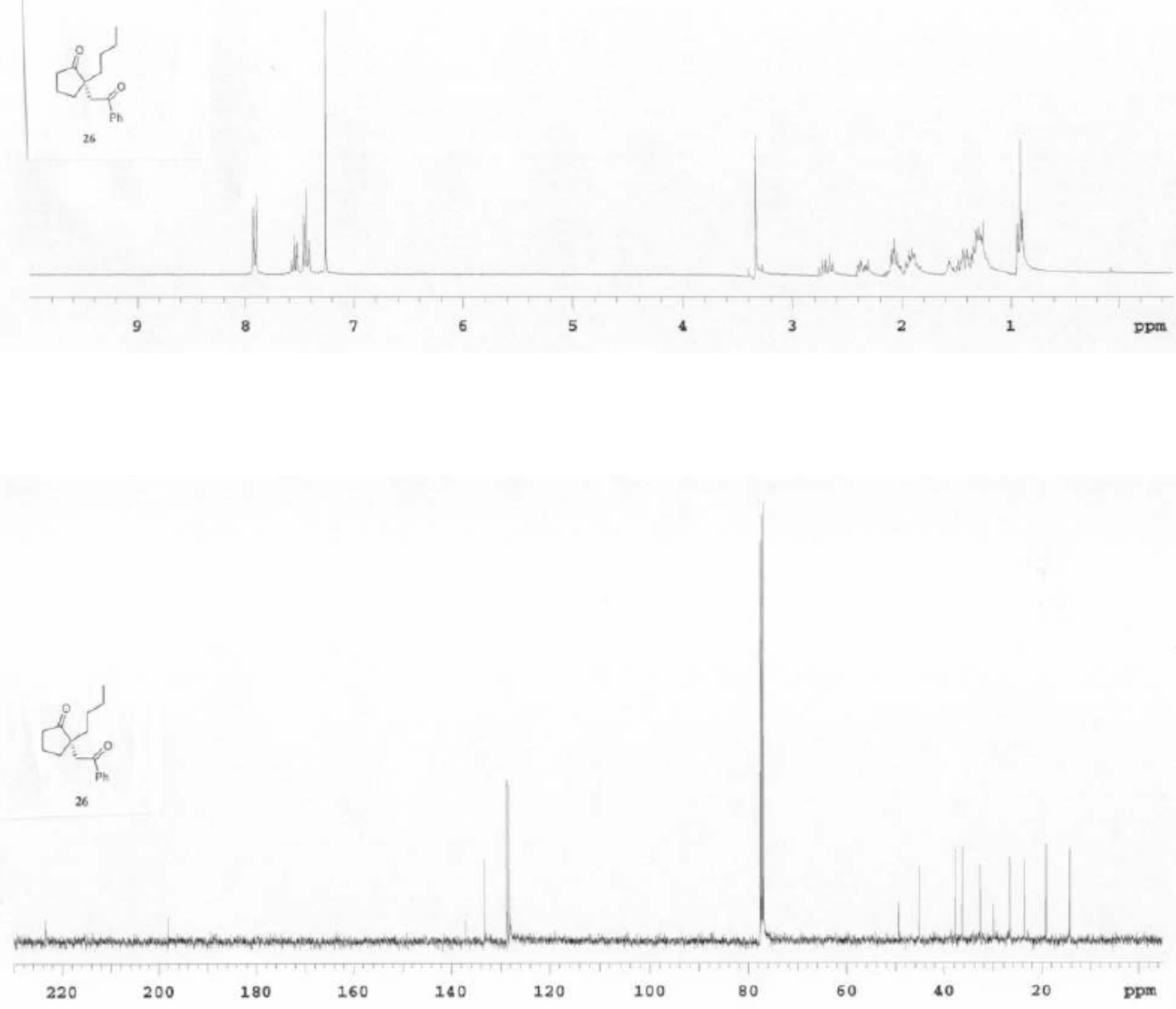